\title{
Neuroimaging Features and Predictors of Outcome in Eclamptic Encephalopathy: A Prospective Observational Study
}

\author{
V. Junewar, R. Verma, P.L. Sankhwar, R.K. Garg, M.K. Singh, H.S. Malhotra, P.K. Sharma, and A. Parihar
}

\begin{abstract}
BACKGROUND AND PURPOSE: Posterior reversible encephalopathy syndrome is associated with eclampsia. We assessed the distribution and nature of typical and atypical cranial MR imaging findings in these patients and their correlation with clinical and laboratory data and predictors of outcome.
\end{abstract}

MATERIALS AND METHODS: Forty-five clinically confirmed cases of eclampsia were included in this prospective observational study. Subjects with hemolysis, elevated liver enzymes, and low platelets syndrome $(n=9)$ and pre-existing neurologic conditions ( 1 with cerebral solitary cysticercus granuloma) were excluded. Patients underwent blood investigations and cranial MR imaging.

RESULTS: Twenty-seven patients had abnormal while 8 had normal MR imaging findings. Involvement of brain regions was as follows: frontal, $88.89 \%$; temporal, $44.44 \%$; parietal, $100 \%$; occipital, $100 \%$; deep gray matter, $29.63 \%$; cerebellum, $22.22 \%$; brain stem, $14.81 \%$. Cytotoxic edema was present in $33.33 \%$ of cases; $66.67 \%$ of patients had mild posterior reversible encephalopathy syndrome; $25.92 \%$ had moderate posterior reversible encephalopathy syndrome; and $7.41 \%$ had severe posterior reversible encephalopathy syndrome. Abnormal neuroimaging findings were significantly associated with altered sensorium; visual disturbances; status epilepticus; and elevated serum creatinine, uric acid, and lactate dehydrogenase $(P=.006, P=.018, P=.015, P=.019, P=.003$, and $P=.001$, respectively). Serum creatinine, uric acid, and lactate dehydrogenase values and the presence of moderate or severe posterior reversible encephalopathy syndrome were significantly associated with mortality $(P<.001, P<.001, P=.009$, and $P=.027$, respectively).

CONCLUSIONS: Neuroimaging in eclampsia demonstrates a higher incidence of atypical distributions and cytotoxic edema than previously thought. Altered sensorium; visual disturbances; status epilepticus; and elevated serum uric acid, lactate dehydrogenase, and creatinine are associated with abnormal neuroimaging findings. Higher serum creatinine, uric acid, and lactate dehydrogenase levels and moderate and severe forms of posterior reversible encephalopathy syndrome are possible predictors of poor outcome.

ABBREVIATIONS: HELLP = hemolysis, elevated liver enzymes, and low platelets; LDH = lactate dehydrogenase; PRES = posterior reversible encephalopathy syndrome; $U A=$ uric acid

$\mathrm{H}$ ypertensive disorders are the most common medical complications of pregnancy, with a reported incidence between $6 \%$ and $8 \%,{ }^{1}$ and they are an important cause of severe morbidity and mortality among mothers and babies. In Africa and Asia, hypertensive disorders of pregnancy, especially pre-eclampsia and eclampsia, are associated with nearly one-tenth of all maternal deaths. ${ }^{2}$

Eclampsia is defined as the occurrence of seizures that cannot be attributed to other causes in a woman with pre-eclampsia. ${ }^{1}$ The

Received December 13, 2013; accepted after revision January 23, 2014.

From the Departments of Neurology (V.J., R.V., R.K.G., M.K.S., H.S.M., P.K.S.), Obstetrics and Gynecology (P.L.S.), and Radiodiagnosis (A.P.), King George's Medical University, Lucknow, India.

Please address correspondence to Rajesh Verma, DM, DNB, Department of Neurology, King George's Medical University, Lucknow, India; e-mail:

drrajeshverma32@yahoo.com

http://dx.doi.org/10.3174/ajnr.A3923

neurologic manifestations of eclampsia are similar to those of hypertensive encephalopathy. ${ }^{3,4}$ Neuroimaging in these patients shows edema of the cortex and subcortical white matter predominantly involving the occipital lobes. There is complete resolution of clinical features and most or all radiologic findings at the normalization of the patient's blood pressure, which can be achieved with administration of antihypertensive drugs and/or delivery of the child and placenta. ${ }^{4}$ This clinicoradiologic entity that regresses in a few weeks with elimination of etiologic factors has been previously termed "hypertensive encephalopathy," "reversible posterior leukoencephalopathy syndrome," 5 "reversible posterior cerebral edema syndrome," 6 and "posterior reversible encephalopathy syndrome" (PRES). ${ }^{7}$ PRES has now become the preferred term, ${ }^{8}$ though to some extent, it is a misnomer because PRES is not always limited to the posterior region of the brain and is not always reversible. Beyond eclampsia, PRES can be associ- 
ated with hypertension due to other causes, immunosuppressant therapy (eg, cyclosporine A), antineoplastic chemotherapy, autoimmune diseases (eg, lupus), infections, sepsis, and shock. ${ }^{5,9}$

Most studies conducted on the neuroradiologic aspects of eclampsia have had small sample sizes. Additionally, although there are studies that correlate various clinical and laboratory parameters with abnormalities on neuroimaging, ${ }^{4,8,10}$ only a few studies ${ }^{11}$ provide information about predictors of outcome in eclamptic encephalopathy. The purpose of this study was to determine the spectrum of MR imaging findings in patients with eclampsia and to evaluate their correlation with clinical and laboratory data. We also sought to determine different predictors of outcome of eclamptic encephalopathy.

\section{MATERIALS AND METHODS}

This was a hospital-based prospective observational study conducted from October 2011 to September 2013 and approved by our institutional ethics committee. Clinically confirmed cases of eclampsia from the obstetrics and gynecology department of the hospital that were referred to the neurology department for opinions were included in the study. The following criteria of the American College of Obstetricians and Gynecologists were used for the diagnosis of eclampsia: 1) systolic blood pressure of $\geq 140$ $\mathrm{mm} \mathrm{Hg}$ or diastolic blood pressure of $\geq 90 \mathrm{~mm} \mathrm{Hg}$ that occurs after 20 weeks of gestation in a previously normotensive female, 2) proteinuria of $\geq 0.3 \mathrm{~g}$ in a 24 -hour urine sample, and 3) $\geq 1$ episode of seizures. ${ }^{1,12}$ Subjects diagnosed as having hemolysis, elevated liver enzymes, and low platelets syndrome (HELLP, according to Tennessee criteria) ${ }^{13}$ were excluded from the study because hematologic abnormalities associated with it might confound with the interpretation of laboratory data. Those patients with pre-existing neurologic conditions were also excluded from the study. All patients were interviewed, examined, and investigated after written informed consent was obtained.

The blood pressure noted just before the onset of neurologic symptoms was recorded. For those patients who were not admitted to the hospital when the neurologic symptoms started, the blood pressure recordings obtained at admission were used. All patients underwent routine blood investigations, including hemoglobin, hematocrit, mean corpuscular volume, total leukocyte count, platelet count, lactate dehydrogenase (LDH), aspartate transaminase, alanine transaminase, serum creatinine, blood urea, and serum uric acid (UA) levels. Cranial MR imaging of all patients was performed between the first and seventh day of the onset of clinical symptoms in a $1.5 \mathrm{~T}$ system. The standard protocol consisted of T1-weighted spin-echo and T2-weighted spinecho and FLAIR sequences along with DWI and ADC maps. T2*weighted imaging was included to detect hemorrhage. Cerebral venous thrombosis was ruled out by using MR venography. None of the patients received intravenous contrast.

\section{Localization of Lesions and Description}

MR imaging of all the cases was screened by 2 experienced neuroradiologists blinded to clinical data, and a consensus review was obtained. Cases were classified as "MR imaging positive" or "MR imaging negative," depending on presence or absence of abnormalities. MR imaging-positive cases were categorized according to the involvement of the following locations: frontal, temporal, parietal, occipital, brain stem, basal ganglia, thalamus, cerebellum, corpus callosum, and periventricular region. In addition, the presence of restricted diffusion was noted. On FLAIR and T2*weighted images, intracranial hemorrhages were noted and further categorized as microhemorrhage $(<5 \mathrm{~mm})$, intraparenchymal hemorrhage ( $>5 \mathrm{~mm}$ ), or subarachnoid hemorrhage.

\section{Severity of PRES}

Cases were classified for severity of PRES on the basis of the extent of hyperintensity, mass effect, and signs of herniation and involvement of atypical locations by using FLAIR imaging ${ }^{14}$ :

Mild: Cortical/subcortical white matter signal-intensity alterations without involvement of periventricular white matter and without mass effect, or involvement of none or only one of the following: cerebellum, brain stem, or basal ganglia.

Moderate: Edema involving the cortex and subcortical white matter without involvement of the periventricular white matter, with mild mass effect but without midline shift/herniation, or involvement of 2 of the following: cerebellum, brain stem, or basal ganglia.

Severe: Edema extending from the cortex to the periventricular white matter or the presence of midline shift/herniation, or involvement of all 3 of the following: cerebellum, brain stem, and basal ganglia.

\section{Treatment}

All patients received magnesium sulfate by the Pritchard regimen immediately at the time of admission. The regimen was aborted with the appearance of signs of toxicity (absent knee jerks, respiratory rate $<16 / \mathrm{min}$, or urine output $<25 \mathrm{~mL} / \mathrm{hr}$ ). ${ }^{15}$ Phenytoin (loading dose of $20 \mathrm{mg} / \mathrm{kg}$ intravenously for 30 minutes followed by $100 \mathrm{mg}$ every 8 hours) was given in such patients if they continued to have seizures. Phenytoin was continued for 3 months after discharge and was then tapered off. ${ }^{16}$

Blood pressure control was achieved with labetalol (20-mg intravenous bolus dose followed by $40 \mathrm{mg}$ if not effective within 10 minutes; then $80 \mathrm{mg}$ every 10 minutes to a maximum total dose of $220 \mathrm{mg}) .{ }^{1,12}$

All patients underwent emergency termination of pregnancy by the obstetrician. ${ }^{17}$ Cesarean delivery was performed only for obstetric indications; the rest of the patients underwent vaginal delivery, either spontaneously or with induction of labor by using intracervical dinoprostone.

Clinical follow-up was at 1- and 3-month intervals after discharge. Outcome was assessed in the form of disability and death by using the modified Rankin scale. Multiple parameters (including demographic data, symptoms at presentation, examination findings, laboratory data, and neuroimaging features) were studied to determine possible predictors of outcome.

Data analysis was performed by using the Statistical Package for the Social Sciences, Version 16 (IBM, Armonk, New York). Discrete data were reported as frequency and percentages, and continuous data were reported as mean $\pm \mathrm{SD}$. In univariate analysis, the Student $t$ test was applied for continuous variables and the Fisher exact test was applied for discrete variables. In multivariate analysis, ordinal logistic regression and binary logistic re- 


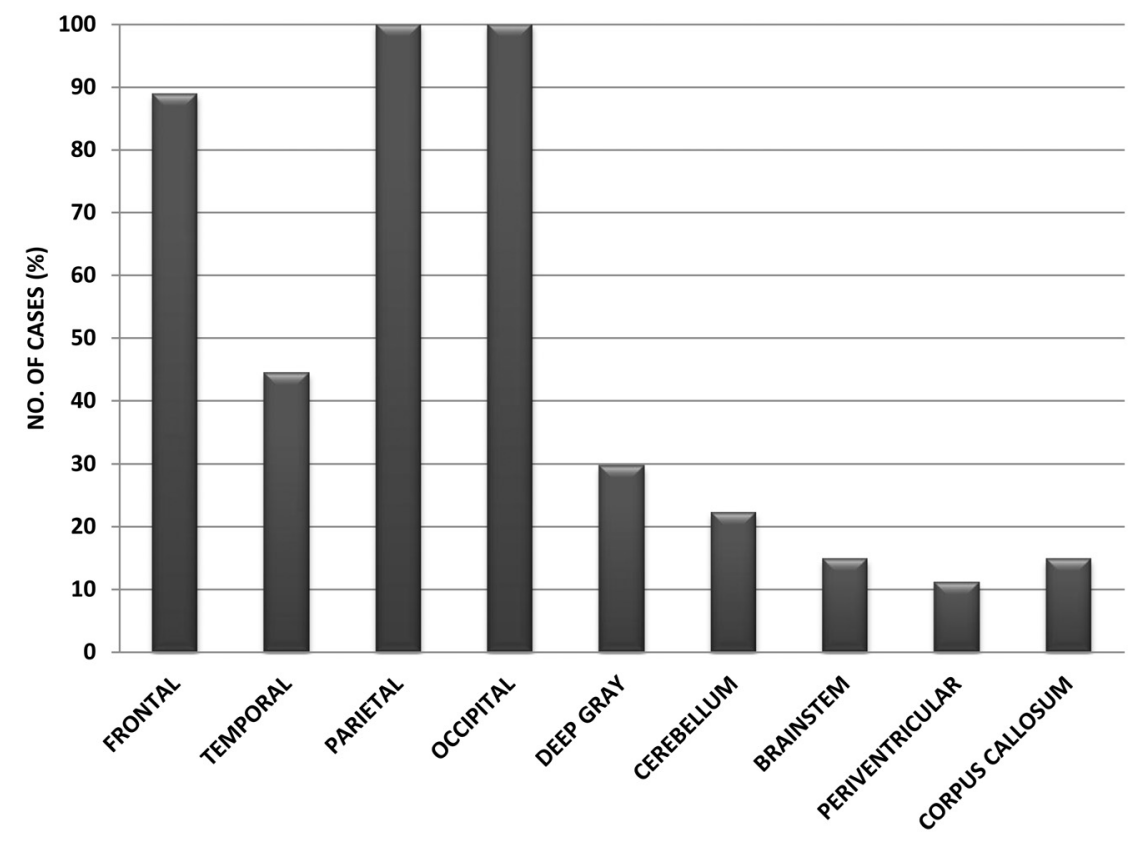

FIG 1. Distribution of lesions in MR imaging-positive cases.

Table 1: Correlation of various parameters with severity of PRES

\begin{tabular}{lccc}
\hline & \multicolumn{2}{c}{ 95\% Confidence Interval } & \\
\cline { 2 - 3 } \multicolumn{1}{c}{ Parameters } & Lower & Upper & P Value \\
\hline Systolic blood pressure & -0.09 & 0.07 & .87 \\
Diastolic blood pressure & -0.08 & 0.14 & .56 \\
Serum UA & -0.19 & 0.83 & .22 \\
Serum LDH & 0.00 & 0.02 & .26 \\
Serum creatinine & -3.12 & 2.33 & .78 \\
\hline
\end{tabular}

gression were used to correlate the severity of PRES and outcome with different parameters, respectively. A $P$ value $<.05$ was considered statistically significant.

\section{RESULTS}

Forty-five patients were enrolled. Ten patients were excluded from the study, 9 for HELLP syndrome and 1 for a solitary cysticercus granuloma on her brain MR imaging. Analysis of the remaining 35 cases follows.

Most of the patients (91.43\%) belonged to the 20- to 29-year age group. Thirty cases $(85.71 \%)$ were primigravida, whereas 5 (14.29\%) were multigravida. Apart from seizures, symptoms included altered sensorium (94.29\%), headache (68.57\%), visual disturbances $(28.57 \%)$, and focal neurologic deficit $(8.57 \%)$. Thirteen $(37.14 \%)$ patients had status epilepticus. Twenty-eight $(80 \%)$ had antepartum eclampsia, $5(14.29 \%)$ had early postpartum eclampsia (first 48 hours postpartum), and 2 (5.71\%) had late postpartum eclampsia ( 48 hours' to 4 weeks' postpartum).

Most patients (32 of 35) underwent MR imaging within 3 days of the onset of neurologic symptoms. Twenty-seven $(77.14 \%)$ cases were MR imaging-positive, while 8 (22.86\%) cases were MR imaging-negative. In the MR imaging-positive cases, the frontal region was involved in $24(88.89 \%)$ cases; the temporal, in 12 (44.44\%) cases; and parietal and occipital regions, in all 27 (100\%) cases. The deep gray matter was involved in $8(29.63 \%)$ cases; the cerebellum, in $6(22.22 \%)$ cases; the brain stem, in 4
(14.81\%) cases; the periventricular region, in 3 (11.11\%) cases; and the corpus callosum, in $4(14.81 \%)$ cases (Fig 1). Hemorrhages were present in 6 $(22.22 \%)$ cases. Five had intraparenchymal hemorrhages (3 lobar, 1 pontine, and 1 caudate), 1 had microhemorrhages, and none had subarachnoid hemorrhage; $33.33 \%$ showed the presence of cytotoxic edema. MR venography did not reveal any abnormality in any patient.

When analyzed for severity, of $27 \mathrm{MR}$ imaging-positive cases, 18 (66.67\%) had mild PRES, 7 (25.92\%) had moderate PRES, and 2 (7.41\%) had severe PRES. However, neither blood pressure nor any of the laboratory parameters (serum UA, serum LDH, and serum creatinine levels) had any significant correlation with the severity of PRES (Table 1).

When MR imaging-positive patients were compared with MR imaging-negative patients, there was no statistically significant difference between them in terms of mean age and frequency of the presence of headache, focal neurologic deficit, and edema. However, the difference was found to be statistically significant for altered sensorium $(P=.006)$, visual disturbances $(P=.018)$, and the presence of status epilepticus $(P=.015)$. The difference among systolic, diastolic, and mean blood pressure was also statistically insignificant. Values of serum creatinine, serum UA, and serum LDH, however, were significantly higher in MR imaging-positive cases $(P=.019, P=.003$, and $P=.001$, respectively) (Table 2).

Twenty-nine patients survived and were discharged from the hospital within 1-2 weeks. Three patients had hemiparesis on presentation; 2 of them had intracerebral hemorrhage and died during the hospital stay. The remaining patient had infarct in the middle cerebral artery territory due to vasospasm, which was demonstrated on MR angiography. She was discharged with mild disability. At 1-month follow-up, there was complete recovery in her disability. Thus, all 29 surviving patients had full recovery (ie, mRS grade 0 ) at the end of 1 month. Six patients died during the hospital stay. Of these, 2 patients died of intraparenchymal hemorrhages (pontine and caudate), 2 had severe cerebral edema and developed signs of herniation during the course of their illness, one had acute renal failure, and one had acute pulmonary edema.

When baseline parameters of patients who survived and patients who died were compared, there was no statistically significant difference between them in terms of mean age and frequency of the presence of headache; altered sensorium; visual disturbances; focal neurologic deficit; status epilepticus; edema of the feet; and systolic, diastolic, and mean blood pressure. However, it was observed that the mean serum creatinine, serum UA, and serum LDH values were significantly higher in patients who died $(P<$ $.001, P<.001$, and $P=.009$, respectively). In addition, the presence of the moderate or severe form of PRES was significantly more common in these patients $(P=.027)$ (Table 3$)$. On multi- 
Table 2: Comparison of demographic data, clinical features, and laboratory data between MRI-positive and MRI-negative patients

\begin{tabular}{|c|c|c|c|c|c|c|}
\hline & \multirow[b]{2}{*}{ MRI-Positive ( $n=27$ ) } & \multirow[b]{2}{*}{ MRI-Negative $(n=8)$} & \multirow[b]{2}{*}{ OR } & \multicolumn{2}{|c|}{$95 \% \mathrm{Cl}$} & \multirow[b]{2}{*}{$P$ Value } \\
\hline & & & & Lower & Upper & \\
\hline Age (yr) & $24.44 \pm 3.53$ & $23.87 \pm 2.47$ & - & -2.16 & 3.30 & .67 \\
\hline Headache & 19 & 5 & 1.42 & 0.27 & 7.44 & 1.00 \\
\hline Altered sensorium & 26 & 4 & 26.0 & 2.29 & 295.65 & $.006^{\mathrm{a}}$ \\
\hline Visual disturbances & 17 & 1 & 11.9 & 1.27 & 111.36 & $.018^{\mathrm{a}}$ \\
\hline Focal deficit & 3 & 0 & - & - & - & 1.00 \\
\hline Status epilepticus & 13 & 0 & - & - & - & $.015^{\mathrm{a}}$ \\
\hline Edema in feet & 15 & 5 & 0.75 & 0.15 & 3.79 & 1.00 \\
\hline $\mathrm{SBP}(\mathrm{mm} \mathrm{Hg})$ & $164.52 \pm 17.03$ & $153.75 \pm 14.07$ & - & -2.70 & 24.24 & .11 \\
\hline $\mathrm{DBP}(\mathrm{mm} \mathrm{Hg})$ & $102.81 \pm 12.71$ & $98.75 \pm 9.91$ & - & -5.90 & 14.03 & .41 \\
\hline $\mathrm{MBP}(\mathrm{mm} \mathrm{Hg})$ & $123.85 \pm 13.39$ & $117.25 \pm 9.80$ & - & -3.81 & 17.01 & .21 \\
\hline Hematocrit (\%) & $32.33 \pm 6.76$ & $32.58 \pm 6.49$ & - & -5.73 & 5.25 & .93 \\
\hline $\mathrm{MCV}(\mathrm{fL})$ & $88.97 \pm 6.02$ & $91.1 \pm 6.17$ & - & -7.08 & 2.83 & .39 \\
\hline $\operatorname{TLC}\left(10^{9} / \mathrm{L}\right)$ & $7.83 \pm 2.23$ & $8.41 \pm 2.00$ & - & -2.37 & 1.20 & .51 \\
\hline Plt $\left(10^{5} / \mathrm{mm}^{3}\right)$ & $1.85 \pm 0.49$ & $2.00 \pm 0.29$ & - & -0.52 & 0.22 & .42 \\
\hline AST (IU/L) & $47.15 \pm 17.83$ & $53.13 \pm 15.22$ & - & -20.15 & 8.20 & .40 \\
\hline ALT (IU/L) & $46.48 \pm 16.12$ & $53.13 \pm 16.14$ & - & -19.85 & 6.56 & .32 \\
\hline Sr. Creatinine (mg/dL) & $1.11 \pm 0.41$ & $0.74 \pm 0.14$ & - & 0.07 & 0.67 & $.019^{a}$ \\
\hline Sr. UA (mg/dL) & $9.47 \pm 2.75$ & $6.31 \pm 0.76$ & - & 1.14 & 5.17 & $.003^{\mathrm{a}}$ \\
\hline Sr. LDH (IU/L) & $458.03 \pm 107.94$ & $319.50 \pm 20.50$ & - & 59.69 & 217.39 & $.001^{\mathrm{a}}$ \\
\hline
\end{tabular}

Note:-SBP indicates systolic blood pressure; DBP, diastolic blood pressure; MBP, mean blood pressure; MCV, mean corpuscular volume; TLC, total leukocyte count; Plt, platelet count; AST, aspartate transaminase; ALT, alanine transaminase; Sr., serum.

a Significant.

Table 3: Comparison of demographic data, clinical features, and laboratory data of cases with respect to outcome

\begin{tabular}{|c|c|c|c|c|c|c|}
\hline & \multirow[b]{2}{*}{ Survived $(n=29)$} & \multirow[b]{2}{*}{ Deceased $(n=6)$} & \multirow[b]{2}{*}{ OR } & \multicolumn{2}{|c|}{$95 \% \mathrm{Cl}$} & \multirow[b]{2}{*}{$P$ Value } \\
\hline & & & & Lower & Upper & \\
\hline Age (yr) & $23.86 \pm 2.76$ & $26.50 \pm 4.93$ & - & -5.55 & 0.27 & .07 \\
\hline Headache & 19 & 5 & 0.38 & 0.04 & 3.713 & .64 \\
\hline Altered sensorium & 24 & 6 & - & - & - & .56 \\
\hline Visual disturbances & 14 & 4 & 0.47 & 0.07 & 2.96 & .66 \\
\hline Focal deficit & 1 & 2 & 0.07 & 0.01 & 0.98 & .07 \\
\hline Status epilepticus & 12 & 1 & 3.53 & 0.36 & 34.19 & .38 \\
\hline Edema in feet & 16 & 4 & 0.62 & 0.10 & 3.91 & .68 \\
\hline SBP (mm Hg) & $159.72 \pm 17.52$ & $173.33 \pm 5.16$ & - & -28.45 & 1.23 & .07 \\
\hline $\mathrm{DBP}(\mathrm{mm} \mathrm{Hg})$ & $100.55 \pm 11.35$ & $108.33 \pm 14.72$ & - & -18.66 & 3.10 & .16 \\
\hline MBP (mm Hg) & $120.76 \pm 12.79$ & $130 \pm 10.95$ & - & -20.76 & 2.19 & .11 \\
\hline Hematocrit (\%) & $32.76 \pm 6.42$ & $30.53 \pm 7.79$ & - & -3.82 & 8.31 & .46 \\
\hline $\mathrm{MCV}(\mathrm{fL})$ & $89.94 \pm 5.86$ & $87.12 \pm 6.84$ & - & -2.67 & 8.32 & .30 \\
\hline $\operatorname{TLC}\left(10^{9} / \mathrm{L}\right)$ & $8.02 \pm 2.31$ & $7.65 \pm 1.33$ & - & -1.62 & 2.38 & .70 \\
\hline Plt $\left(10^{5} / \mathrm{mm}^{3}\right)$ & $1.88 \pm 0.49$ & $1.88 \pm 0.20$ & - & -0.41 & 0.43 & .96 \\
\hline AST (IU/L) & $50.17 \pm 18.09$ & $40.50 \pm 9.75$ & - & -5.93 & 25.27 & .22 \\
\hline ALT (IU/L) & $47.66 \pm 17.44$ & $49.67 \pm 7.99$ & - & -16.94 & 12.92 & .79 \\
\hline Sr. Creatinine (mg/dL) & $0.88 \pm 0.26$ & $1.72 \pm 0.07$ & - & -1.06 & .62 & $<.001^{\mathrm{a}}$ \\
\hline Sr. UA (mg/dL) & $7.89 \pm 1.54$ & $12.87 \pm 3.78$ & - & -6.84 & -3.10 & $<.001^{\mathrm{a}}$ \\
\hline Sr. LDH (IU/L) & $404.44 \pm 109.06$ & $532.33 \pm 44.68$ & - & -220.90 & -34.85 & $.009^{\mathrm{a}}$ \\
\hline Positive MRI findings & 21 & 6 & - & - & - & .30 \\
\hline Moderate and severe PRES & 5 & 4 & 0.10 & 0.02 & 0.73 & $.027^{\mathrm{a}}$ \\
\hline Cytotoxic edema & 7 & 2 & 0.64 & 0.10 & 4.25 & .64 \\
\hline
\end{tabular}

Note:-SBP indicates systolic blood pressure; DBP, diastolic blood pressure; MBP, mean blood pressure; MCV, mean corpuscular volume; TLC, total leukocyte count; Plt, platelet count; AST, aspartate transaminase; ALT, alanine transaminase; Sr., serum.

a Significant.

variate analysis, however, we could not find any statistically significant correlation between outcome and any of these factors.

\section{DISCUSSION}

Eclampsia is one of the many causes of PRES. MR imaging of the brain in PRES is characterized by areas of altered signal intensity predominantly involving the occipital and parietal regions. The lesions are usually isointense to hypointense on T1WI and hyperintense on T2WI and FLAIR sequences. There is more involvement of white matter than gray matter, which is consistent with vasogenic edema. Lesions are mostly hemispheric and bilaterally symmetric. ${ }^{18}$ Lesions with similar characteristics were detected in all of our MR imaging-positive cases. With more severe disease, extensive involvement of the brain occurs along with involvement of atypical areas like the frontal lobes, temporal lobes, corpus callosum, cerebellum, brain stem, basal ganglia, and thalami (Figs 2-4). ${ }^{18}$ Recently, it has been shown that the anterior regions are more commonly involved than previously observed. Various studies have shown that the range of frontal lobe involvement varies widely from $30 \%$ to $78.9 \% .^{4,14,19}$ In our study, the frontal 


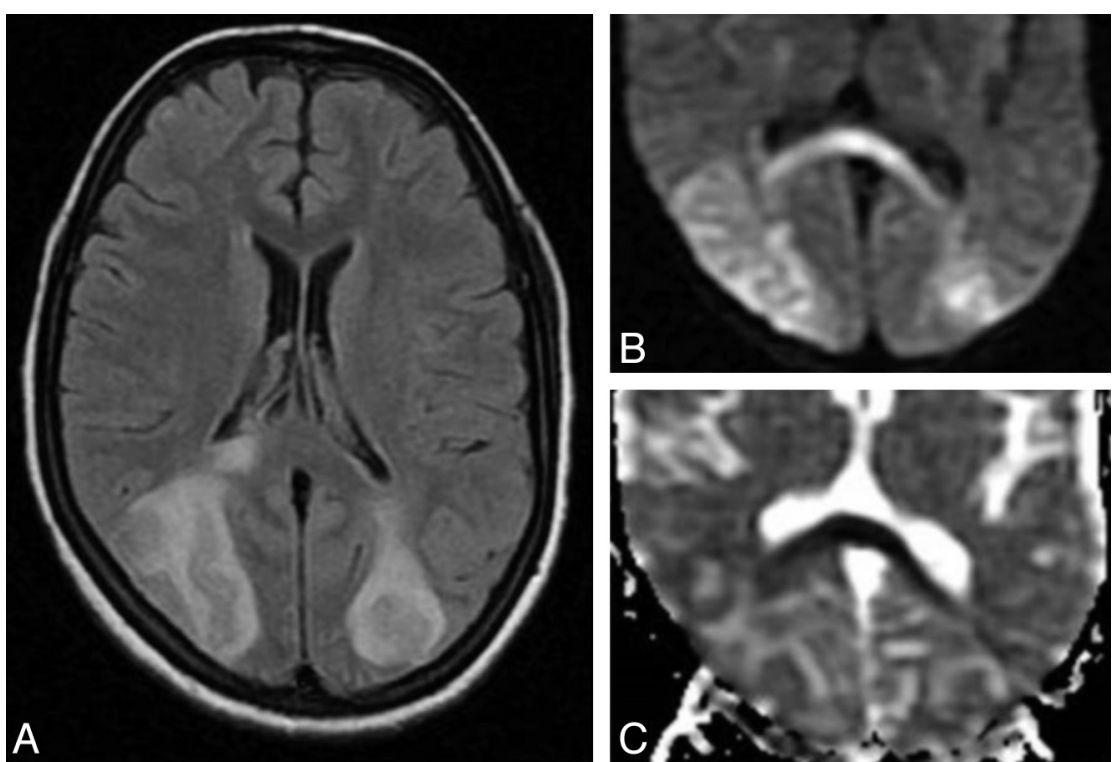

FIG 2. MR images of the brain of a 25 -year-old woman with antepartum eclampsia. A, FLAIR MR image shows edema involving the cortex and subcortical white matter of the bilateral occipital and parietal regions and the splenium of the corpus callosum. $B$, DWI shows hyperintensity in corresponding areas. $C, A D C$ image shows hyperintensity in the bilateral occipital and parietal regions but hypointensity in the splenium of the corpus callosum.
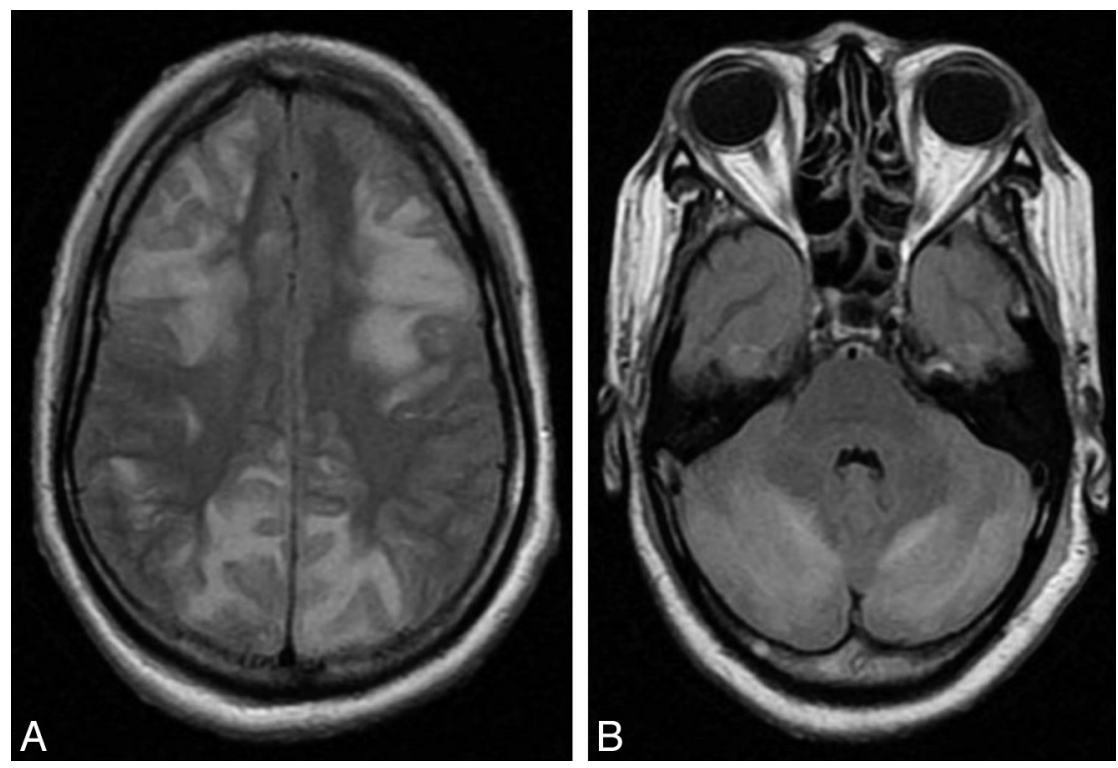

FIG 3. FLAIR MR images of the brain of a 19-year-old woman with antepartum eclampsia showing involvement of bilateral frontal, parietal $(A)$, and cerebellar $(B)$ regions.

lobe was involved in $88.89 \%$ of cases, thus reinforcing the thought that anterior regions are also frequently involved in PRES.

Hemorrhages in PRES may be seen in as many as $15 \%$ of cases. ${ }^{20}$ Hefzy et $\mathrm{al}^{21}$ found 3 types of hemorrhages, namely, microhemorrhages, intraparenchymal hemorrhages, and subarachnoid hemorrhages, with equal frequency. Susceptibility-weighted imaging has increased the ability to detect microhemorrhages. They can be seen in more than half of patients. ${ }^{22}$ In our study, 6 (22.22\%) patients had hemorrhagic lesions. Two of these patients died; one had pontine hemorrhage and the other had caudate hemorrhage. In terms of the presence of hemorrhage, the difference between the surviving (4/29) and deceased (2/6) patients was statistically insignificant $(P=.58)$. Therefore, it seems that the site of hemorrhage rather than just its presence is more important.

In our study, 8 (22.86\%) patients had normal brain imaging findings. A recent study by Brewer et $\mathrm{al}^{23}$ found PRES in $97.9 \%$ of patients with eclampsia by using $\geq 1$ technique of imaging. They could find PRES only in $87.2 \%$ of patients by using MR imaging without contrast, however, which is close to the case positivity in our study (77.14\%). MR imaging findings in eclampsia may be normal, especially if the syndrome had a short duration. ${ }^{19}$ The clinical course of MR imaging-negative patients was more benign because they had less frequent headaches and visual disturbances; none of them had status epilepticus and none died.

DWI helps to differentiate vasogenic edema of PRES from cytotoxic edema. ${ }^{24}$ Focal areas of restricted diffusion (ADC dark, DWI bright) may be seen in PRES, however (Figs 2 and 4). ${ }^{19,24}$ This finding is believed to be due to compromised tissue microcirculation, either secondary to the mass effect of vasogenic edema or due to reactionary vasoconstriction. ${ }^{14,25}$ Although cases with restricted diffusion have been well-reported, we found that cytotoxic edema is more commonly seen than was previously thought. In our study, cytotoxic cerebral edema was seen in $33.33 \%$ of patients compared with $17.3 \%$ of patients in the study by McKinney et al in 2007. ${ }^{14}$ This higher frequency of cytotoxic edema in eclampsia is probably due to reactionary vasoconstriction. Reversible segmental narrowing of large and medium-sized cerebral arteries had been well documented previously in patients with eclampsia on conventional angiography and MR angiography. A high level of endothelin, a potent vasoconstrictor peptide seen in patients with eclampsia, might be responsible for the vasospasm. ${ }^{26}$

The exact pathogenesis of PRES is not yet completely understood. Extravasation of fluid in the parenchyma is thought to be a major causative factor. ${ }^{8}$ This might be related to the disturbance of the cerebral autoregulation mechanism or endothelial dysfunction. ${ }^{4,8,25}$ In PRES with hypertension, dysfunction of cerebral autoregulation could be responsible for serum extravasation. However, PRES does occur without hypertension. It is proposed that direct endothelial dysfunction leading to an increase in the permeability of the blood-brain barrier may be the additional mech- 

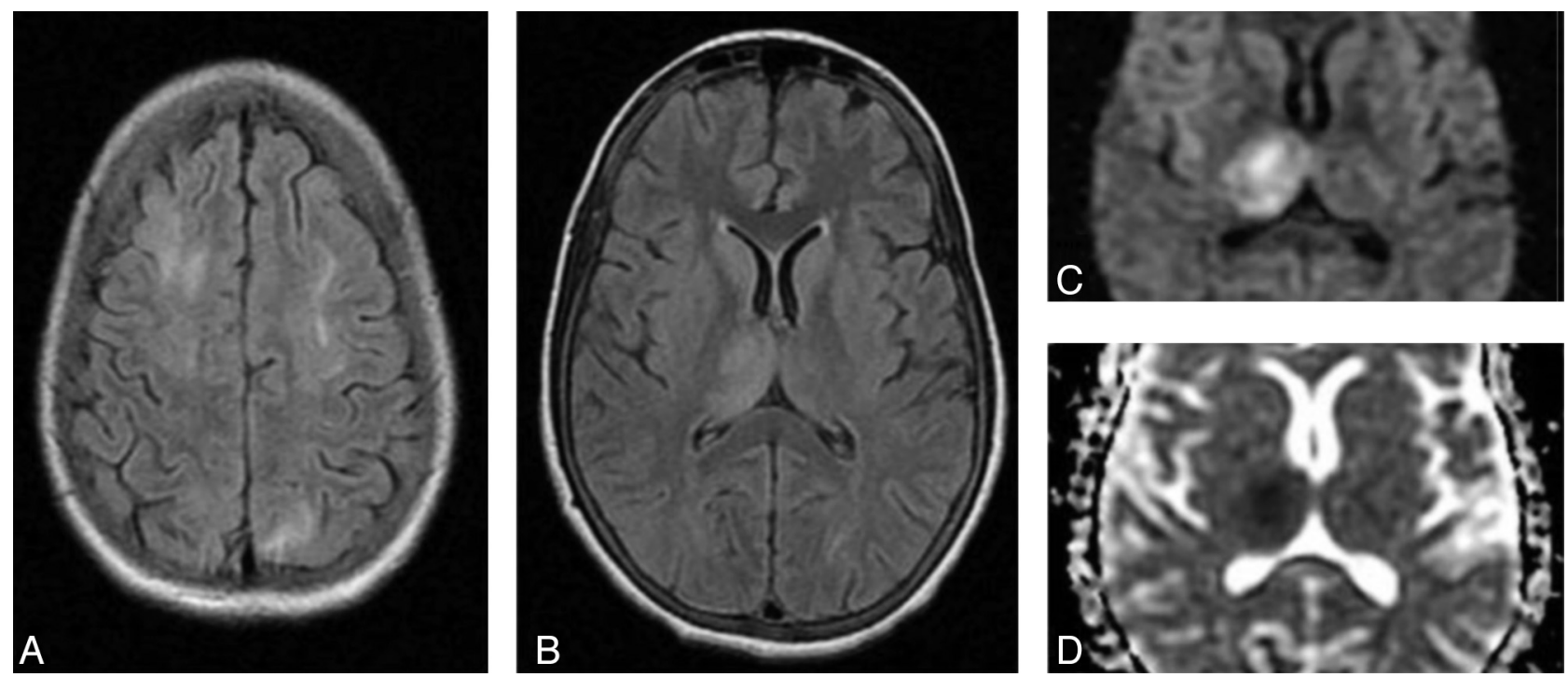

FIG 4. Brain MR images of a 20-year-old woman with early postpartum eclampsia. A, FLAIR MR image shows edema involving the bilateral frontal and parietal regions. FLAIR (B), DWI (C), and ADC (D) show involvement of the right thalamus with cytotoxic edema.

anism responsible for parenchymal fluid extravasation. ${ }^{4,8} \mathrm{Al}-$ though the exact cause of the endothelial injury in patients with eclampsia is not known, circulating endothelial toxins or antibodies are thought to be responsible. ${ }^{4}$

In our study, abnormal neuroimaging was associated with high serum levels of LDH, which are an indicator of hemolysis. Endothelial injury causes microvascular hemolysis leading to a rise in serum LDH levels. In addition, the brain lesions were found to be associated with high serum UA and serum creatinine levels. Renal dysfunction secondary to renal endothelial injury results in a rise in serum levels of UA and creatinine. In our study, the difference between blood pressure values of patients with or without brain lesions on MR imaging was statistically insignificant. Therefore, it seems that brain lesions were secondary to endothelial injury, rather than a rise in blood pressure. These findings correlate with the findings of Schwartz et $\mathrm{al}^{4}$ and Demirtaş et al. ${ }^{8}$

Recently Gao et $\mathrm{al}^{10}$ showed the relation of serum levels of LDH with the degree of cerebral edema. However, we did not find any significant correlation of the severity of PRES with any biomarker of endothelial dysfunction. We postulate that eclampsia, being a multisystem disorder, is associated with widespread endothelial dysfunction. These markers are not specific for the cerebral vascular endothelium. Hence, their elevation suggests just diffuse endothelial dysfunction, and absolute values may not correlate with the degree of cerebral endothelial dysfunction and thus with the severity of cerebral edema. Therefore, more studies are required aiming at correlation between certain specific biomarkers of cerebral vascular endothelium and the severity of PRES.

To date, only a few studies ${ }^{11}$ have commented on predictors of outcome in eclampsia. In our study, we found that the mean serum creatinine, $\mathrm{UA}$, and $\mathrm{LDH}$ values were significantly higher in patients who died. In addition, the presence of the moderate or severe form of PRES was significantly higher in these patients as opposed to the mild form of PRES, which was more common in surviving patients.

\section{CONCLUSIONS}

Neuroimaging in eclampsia demonstrates a higher incidence of atypical distribution and the presence of cytotoxic edema than previously thought. Altered sensorium, visual disturbances, presentation with status epilepticus, and elevated serum UA, LDH, and creatinine levels are more likely to be associated with abnormal neuroimaging findings. Additionally, higher serum creatinine, UA, and LDH levels and moderate and severe forms of PRES on neuroimaging are possible predictors of poor outcome.

\section{REFERENCES}

1. Report of the National High Blood Pressure Education Program Working Group on high blood pressure in pregnancy. Am J Obstet Gynecol 2000;183:S1-22

2. World Health Organization. WHO Recommendations for Prevention and Treatment of Pre-Eclampsia and Eclampsia. Geneva, Switzerland: World Health Organization; 2011

3. Schwartz RB, Jones KM, Kalina P, et al. Hypertensive encephalopathy: findings on CT, MR imaging, and SPECT imaging in 14 cases. AJR Am J Roentgenol 1992;159:379-83

4. Schwartz RB, Feske SK, Polak JF, et al. Preeclampsia-eclampsia: clinical and neuroradiographic correlates and insights into the pathogenesis of hypertensive encephalopathy. Radiology 2000;217:371-76

5. Hinchey J, Chaves C, Appignani B, et al. A reversible posterior leukoencephalopathy syndrome. N Engl J Med 1996;334:494-500

6. Dillon WP, Rowley H. The reversible posterior cerebral edema syndrome. AJNR Am J Neuroradiol 1998;19:591

7. Casey SO, Sampaio RC, Michel E, et al. Posterior reversible encephalopathy syndrome: utility of FLAIR imaging in the detection of cortical and subcortical lesions. AJNR Am J Neuroradiol 2000;21:1199-206

8. Demirtaş O, Gelal F, Vidinli BD, et al. Cranial MR imaging with clinical correlation in preeclampsia and eclampsia. Diagn Interv $R a-$ diol 2005;11:189-94

9. Petrovic BD, Nemeth AJ, McComb EN, et al. Posterior reversible encephalopathy syndrome and venous thrombosis. Radiol Clin $\mathrm{N}$ Am 2011;49:63-80

10. Gao B, Liu FL, Zhao B. Association of degree and type of edema in posterior reversible encephalopathy syndrome with serum lactate

AJNR Am J Neuroradiol 35:1728-34 Sep 2014 www.ajnr.org

1733 
dehydrogenase level: initial experience. Eur J Radiol 2012;81: 2844-47

11. Jaiswar SP, Gupta A, Sachan R, et al. Lactic dehydrogenase: a biochemical marker for preeclampsia-eclampsia. J Obstet Gynaecol India 2011;61:645-48

12. ACOG Committee on Practice Bulletins-Obstetrics. ACOG practice bulletin: diagnosis and management of preeclampsia and eclampsia. number 33, January 2002. Obstet Gynecol 2002;99:159-67

13. Haram K, Svendsen E, Abildgaard U. The HELLP syndrome: clinical issues and management: a review. BMC Pregnancy Childbirth 2009;9:8

14. McKinney AM, Short J, Truwit CL, et al. Posterior reversible encephalopathy syndrome: incidence of atypical regions of involvement and imaging findings. AJR Am J Roentgenol 2007;189:904-12

15. Duley L. Magnesium sulphate regimens for women with eclampsia: messages from the Collaborative Eclampsia Trial. $\mathrm{Br} J$ Obstet Gynaecol 1996;103:103-05

16. Chakravarty A, Chakrabarti SD. The neurology of eclampsia: some observations. Neurol India 2002;50:128-35

17. Sibai BM. Diagnosis and management of gestational hypertension and preeclampsia. Obstet Gynecol 2003;102:181-92

18. Feske SK. Posterior reversible encephalopathy syndrome: a review. Semin Neurol 2011;31:202-15

19. Bartynski WS, Boardman JF. Distinct imaging patterns and lesion distribution in posterior reversible encephalopathy syndrome. AJNR Am J Neuroradiol 2007;28:1320-27

20. Bartynski WS. Posterior reversible encephalopathy syndrome. Part 1. Fundamental imaging and clinical features. AJNR Am J Neuroradiol 2008;29:1036-42

21. Hefzy HM, Bartynski WS, Boardman JF, et al. Hemorrhage in posterior reversible encephalopathy syndrome: imaging and clinical features. AJNR Am J Neuroradiol 2009;30:1371-79

22. McKinney AM, Sarikaya B, Gustafson C, et al. Detection of microhemorrhage in posterior reversible encephalopathy syndrome using susceptibility-weighted imaging. AJNR Am J Neuroradiol 2012;33: 896-903

23. Brewer J, Owens MY, Wallace K, et al. Posterior reversible encephalopathy syndrome in $\mathbf{4 6}$ of $\mathbf{4 7}$ patients with eclampsia. Am J Obstet Gynecol 2013;208:468.e1-6

24. Schaefer PW, Buonanno FS, Gonzalez RG, et al. Diffusion-weighted imaging discriminates between cytotoxic and vasogenic edema in a patient with eclampsia. Stroke 1997;28:1082-85

25. Ay H, Buonanno FS, Schaefer PW, et al. Posterior leukoencephalopathy without severe hypertension: utility of diffusion-weighted MRI. Neurology 1998;51:1369-76

26. Sengar AR, Gupta RK, Dhanuka AK, et al. MR imaging, MR angiography, and MR spectroscopy of the brain in eclampsia. AJNR Am J Neuroradiol 1997;18:1485-90 